\title{
Chemotherapy-induced nausea and vomiting in patients with breast cancer: a prospective cohort study
}

\author{
Yoichi Naito ${ }^{1,2}$ (1) $\cdot$ Yuichiro Kai $^{3} \cdot$ Takashi Ishikawa $^{4} \cdot$ Tomoyuki Fujita $^{5} \cdot$ Kanou Uehara $^{6} \cdot$ Hiroyoshi Doihara $^{7}$. \\ Shinya Tokunaga ${ }^{8} \cdot$ Mototsugu Shimokawa $^{9} \cdot$ Yoshinori Ito $^{10} \cdot$ Toshiaki Saeki $^{11}$
}

Received: 22 December 2017 / Accepted: 7 August 2019 / Published online: 12 August 2019

(c) The Author(s) 2019

\begin{abstract}
Purpose To explore the actual status of chemotherapy-induced nausea and vomiting (CINV) through a multicenter prospective cohort study.

Methods Patients with breast cancer treated with moderately emetogenic (MEC) or highly emetogenic (HEC) chemotherapy were eligible. A 7-day diary was provided for all patients. Acute and delayed CINV were defined as nausea and vomiting that developed $\leq 24$ or $>24 \mathrm{~h}$ after the start of chemotherapy, respectively. The severity of nausea was evaluated with a visual analog scale (VAS). We also assessed the accuracy of estimations of CINV by medical staff.

Results In total, 426 patients were included; 352 patients (82.6\%) received HEC, and 74 (17.3\%) received MEC. In the acute phase, $44.9 \%$ of patients receiving HEC and 5.4\% receiving MEC experienced nausea, and $12.8 \%$ receiving HEC and none receiving MEC experienced vomiting. More patients experienced nausea in both groups and vomiting in MEC during the delayed phase (nausea: 59.4\% in HEC and 44.6\% in MEC group; vomiting: 11.1\% in HEC; and $13.5 \%$ in MEC group) than during the acute phase. Estimations of CINV by medical staff were not accurate, with a kappa coefficient of 0.10 and 0.08 for acute nausea and vomiting and 0.02 and 0.01 for delayed. The VAS scores showed that in the HEC group, the degree of nausea was worst on the first day.
\end{abstract}

Conclusions The degree of nausea was worst in the acute phase, although delayed nausea was more in proportion in HEC. Estimation by medical staff is not accurate.

Keywords Chemotherapy-induced nausea and vomiting · Breast cancer · Antiemetics · Guideline

Yoichi Naito

ynaito@east.ncc.go.jp

1 Department of Breast and Medical Oncology, National Cancer Center Hospital East, 6-5-1 Kashiwanoha, Kashiwa, Chiba 277-8577, Japan

2 Department of Developmental Therapeutics, National Cancer Center Hospital East, Kashiwa, Japan

3 Ueo Breast Cancer Hospital, Oita, Japan

4 Department of Breast and Thyroid Surgery, Yokohama City University Medical Center, Yokohama, Kanagawa, Japan

5 Department of Breast Surgery, Tokyo Medical University Ibaraki Medical Center, Ami, Ibaraki, Japan

6 Department of Surgery, Nahanishi Clinic, Naha, Okinawa, Japan
7 Department of Breast and Endocrinological Surgery, Okayama University Hospital, Okayama, Japan

8 Department of Medical Oncology, Osaka City General Hospital, Osaka, Japan

9 Center of Clinical Research, National Kyushu Cancer Center, Fukuoka, Japan

10 Department of Breast Medical Oncology, Breast Oncology Center, Cancer Institute Hospital, Japanese Foundation for Cancer Research, Tokyo, Japan

11 Department of Breast Oncology, International Medical Center, Saitama Medical University, Saitama, Japan 


\section{Introduction}

Breast cancer is one of the most common cancers worldwide $[1,2]$. Survival of patients with breast cancer has dramatically improved because of effective screening system and advances in multimodal therapy, including surgery, radiotherapy, and systemic therapy [3]. Chemotherapy currently plays an important role in both the perioperative period and metastatic setting.

Chemotherapy-induced nausea and vomiting (CINV) is one of the most common and clinically serious adverse reactions to chemotherapy, despite the development of global and domestic guidelines for CINV by the American Society of Clinical Oncology (ASCO) [4], Multinational Association of Supportive Care in Cancer (MASCC)/ European Society of Medical Oncology (ESMO) [5], National Comprehensive Cancer Network (NCCN) [6], and Japanese Society of Clinical Oncology (JSCO). These guidelines consistently recommend the combination of a 5-hydroxytryptamine receptor antagonist (5HT3RA) and dexamethasone for moderately emetogenic chemotherapy (MEC), and a combination of three antiemetics comprising the above two drugs and an aprepitant or fosaprepitant for highly emetogenic chemotherapy (HEC). However, the actual status of clinical practice with respect to these guidelines as well as the proportion and degree of CINV under the optimal antiemetics according to the guidelines are not well studied. Therefore, we conducted a nationwide survey to study the incidence of CINV and the use of antiemetics in accordance with the above guidelines. We additionally assessed estimations of CINV by medical staff to determine the accuracy of CINV predictions by physicians and other medical staff.

\section{Materials and methods}

\section{Study design}

This was a multicenter prospective cohort study. A nationwide survey of CINV was conducted by the CINV Study Group of Japan. We herein present the results of the breast cancer cohort; the results of the entire cohort have been published elsewhere [7]. We selected university hospitals, cancer centers, and cancer treatment hospitals certified by the Ministry of Health, Labour and Welfare (which should serve as core centers for cancer treatment) to participate in this study.

\section{Enrollment of patients}

The inclusion criteria have been described in detail elsewhere [7]. In brief, patients scheduled to undergo MEC or HEC for first-time treatment of cancer were eligible for the current study. Classification of the emetogenic risk of anticancer drugs was based on the Guidelines for Appropriate Use of Antiemetic Drugs, Version 1, published by JSCO [8] and created based on the NCCN Clinical Practice Guidelines in Oncology-Antiemetics-ver. 4, 2009.

To investigate estimation of CINV by medical staff, the staff members were requested to fill out questionnaires regarding their estimation of the severity of symptoms in the acute and delayed phases of CINV on the same registration form if they thought that their patients would develop CINV. Acute and delayed CINV were defined as nausea and vomiting that developed $\leq 24$ or $>24 \mathrm{~h}$ after the start of chemotherapy, respectively.

We aimed to register 450 patients with breast cancer, 700 with gastrointestinal cancer, 200 with gynecological cancer, 200 with hematological malignancies, and 450 with lung cancer.

\section{Patient diary and case reports}

A 7-day diary to record CINV prior to commencement of cancer chemotherapy was provided to each patient. In brief, digestive symptoms such as the development and severity of nausea, frequency of vomiting, amount of food intake, number of salvage treatments, and confirmation of hospitalization or an outpatient visit were recorded. Patients were required to make an entry in the diary every day for 7 days from commencement of anticancer MEC or HEC. The severity of nausea and oral intake were assessed with a linear visual analog scale (VAS; with maximum of $100 \mathrm{~mm}$ ) and a facial scale. The patients were requested to fill in their diaries and send them to the central office in the return envelope provided.

The following patient characteristics were also collected: sex, age, treatment history, use of an anxiolytic drug before administration of the anticancer drug, use of opioids, alcohol intake history, potential risk factors for CINV (history of motion sickness or pregnancy-related vomiting), Eastern Cooperative Oncology Group (ECOG) performance status, complete blood count, blood biochemistry results, cancer chemotherapy regimen, and details of antiemetic therapy and salvage treatment for CINV.

\section{Estimation of CINV by medical staff}

Medical staff estimated whether the patient would develop nausea, vomiting, and anorexia in both acute and delayed phase prior to chemotherapy, mainly based on the patient characteristics such as age, motion sickness, and experience of hyperemesis gravidarum. And if the medical staff 
Fig. 1 Patient flow. In total, 1910 patients were included in the whole study and 429 had breast cancer. One patient treated with cisplatin and irinotecan and two male patients were excluded; therefore, 426 patients were included for the current analyses

\section{Number of registered patients in 5 cancers: 2,068}

\section{Number of HEC or MEC patients' with diary paired with CRF with Staff's report: 1,910}

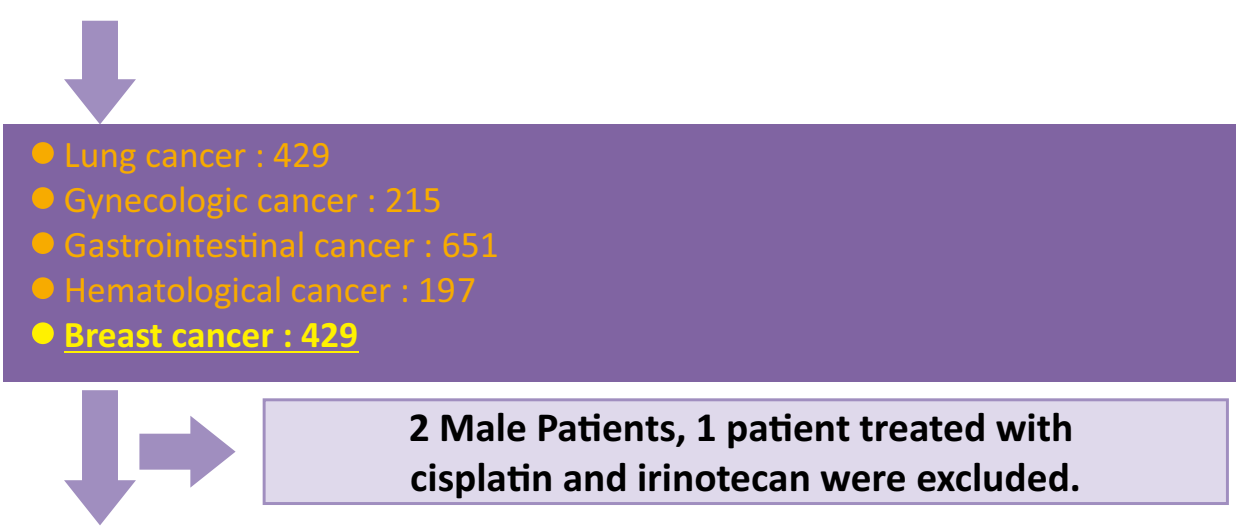

Breast cancer : 426 were analyzed suspected the patient would develop nausea, the degree of nausea was estimated by VAS (scored from 0 to 10 ).

\section{Statistical analysis}

Differences in the occurrence of CINV between patients receiving $\mathrm{HEC}$ or MEC and among risk factors for emesis were analyzed using Fisher's exact test. The numbers of risk factors for CINV in the collected data sets were analyzed by multivariate logistic regression analysis (Wald's test). All reported $p$ values correspond to a two-sided test, and $p$ values of $\leq 0.05$ were considered statistically significant. Analyses were carried out with SAS for Windows, release 9.3 (SAS Institute, Cary, NC).

We excluded one patient treated with cisplatin and irinotecan (CDDP + CPT-11) from analysis as this combination regimen is not standard and widely used in ordinary breast cancer.

\section{Results}

\section{Patients}

In total, 1910 patients were included between April 2011 and December 2012 in the whole study, and 429 had breast cancer. We excluded one patient treated with cisplatin and irinotecan as this regimen is not used widely for breast cancer. And two male patients were also excluded as our analyses included hyperemesis gravidarum as a covariate (Fig. 1). The characteristics of the 426 patients with breast cancer are listed in Table 1. Almost all of these patients had an ECOG
PS of 0 . A total of 352 patients (82.6\%) received HEC and $74(17.3 \%)$ received MEC. The most common HEC regimen was FEC (5-fluorouracil, epirubicin, and cyclophosphamide), and the most common MEC regimen was TC

Table 1 Patient characteristics of breast cancer in the study

\begin{tabular}{|c|c|c|}
\hline & $\begin{array}{l}\text { Number of patients } \\
(n=426)\end{array}$ & $\%$ \\
\hline \multicolumn{3}{|l|}{ Sex } \\
\hline Male & 0 & 0.0 \\
\hline Female & 426 & 100.0 \\
\hline \multicolumn{3}{|l|}{ Stage } \\
\hline 1 & 69 & 16.2 \\
\hline 2 & 241 & 56.6 \\
\hline 3 & 84 & 19.7 \\
\hline 4 & 19 & 4.5 \\
\hline Recurrence & 12 & 2.8 \\
\hline \multicolumn{3}{|l|}{ PS } \\
\hline 0 & 412 & 96.7 \\
\hline 1 & 13 & 3.1 \\
\hline 2 & 1 & 0.2 \\
\hline \multicolumn{3}{|l|}{ HEC $(n=352)$} \\
\hline FEC & 210 & 59.7 \\
\hline $\mathrm{EC}$ & 101 & 28.7 \\
\hline $\mathrm{AC}$ & 41 & 11.6 \\
\hline \multicolumn{3}{|c|}{ HEC prophylactic regimen } \\
\hline 3 antiemetics & 307 & 87.2 \\
\hline 2 antiemetics & 32 & 9.1 \\
\hline Antiemetics other & 13 & 3.7 \\
\hline \multicolumn{3}{|l|}{$\operatorname{MEC}(n=74)$} \\
\hline TC & 62 & 83.8 \\
\hline
\end{tabular}


Table 1 continued

\begin{tabular}{lcr}
\hline & $\begin{array}{l}\text { Number of patients } \\
(n=426)\end{array}$ & $\%$ \\
\hline CBDCA+DOC & 9 & 12.2 \\
CMF & 2 & 2.7 \\
CBDCA + PTX & 1 & 1.4 \\
MEC prophylactic regimen & & \\
3 antiemetics & 3 & 4.1 \\
2 antiemetics & 58 & 78.4 \\
Antiemetics other & 13 & 17.6 \\
\hline
\end{tabular}

$F E C$ fluorouracil + epirubicin + cyclophosphamide, $E C$ epirubicin + cyclophosphamide, $A C$ doxorubicin + cyclophosphamide, $T C$ docetaxel + cyclophosphamide, $C B D C A$ carboplatin, $D O C$ docetaxel, $C M F$ cyclophosphamide + methotrexate + fluorouracil, $P T X$ paclitaxel

(docetaxel and cyclophosphamide). In HEC patients, the proportion of adherence to the JSCO guideline was $87 \%$ and in MEC $82 \%$.

\section{Nausea and vomiting}

Figure 2 shows the proportion of patients with nausea and vomiting. In the acute phase $(\leq 24 \mathrm{~h}$ from administration of emetogenic agents), $44.9 \%$ of patients receiving HEC and $5.4 \%$ of patients receiving MEC experienced nausea, and $12.8 \%$ of those receiving HEC and $0.0 \%$ of those receiving MEC experienced vomiting. In the delayed phase ( $>24 \mathrm{~h}$ from administration), more patients experienced nausea in both groups and vomiting in MEC during the delayed phase (nausea: $59.4 \%$ of those receiving HEC and $44.6 \%$ of those receiving MEC; vomiting: $11.1 \%$ of those receiving HEC and $13.5 \%$ of those receiving MEC) than during the acute phase. The VAS scores (Fig. 3) showed that in patients receiving HEC, the degree of nausea was worst on the first day and gradually improved. Conversely, nausea was sustained without peaking in patients receiving MEC.

\section{Estimation of nausea and vomiting by medical staff}

Table 2 shows the medical staff's estimations of whether patients would develop nausea and vomiting. In the acute phase, $78.5 \%$ of patients ( 335 of 426 ) were estimated to develop nausea; however, 196 patients (46.0\%) did not actually experience nausea. The kappa coefficient was 0.0958 . With regard to vomiting, the kappa coefficient was 0.0795 . In the delayed phase, the kappa coefficient was 0.0237 for nausea and 0.0092 for vomiting.

\section{Analysis of risk factors for nausea and vomiting}

Multivariate analysis revealed that pregnancy experience (odds ratio [OR] 2.14; 95\% confidence interval [CI] $1.32-3.47 ; p=0.0021$ ), treatment with HEC (odds ratio [OR] 18.7; 95\% confidence interval [CI] 6.42-54.21; $p<0.0001$ ), and younger age (odds ratio [OR] for older age as continuous variables, $0.94 ; 95 \%$ confidence interval $[\mathrm{CI}]$ $0.91-0.96 ; p<0.0001)$ were significantly correlated with developing acute-phase nausea (Fig. 4a). Younger age was also significantly correlated with acute-phase vomiting (OR 0.96; 95\% CI, 0.93-0.99; $p=0.0062$ ) (Fig. 4b).

In the delayed phase, experience of motion sickness (OR $3.43 ; 95 \%$ CI $2.16-5.46 ; p<0.0001$ ), younger age (OR 0.98; 95\% CI 0.96-1.00; $p=0.0153)$, a higher serum albumin concentration (OR 2.02; 95\% CI 1.07-3.83; $p=0.0313$ ), and treatment with HEC (odds ratio [OR] 2.04; 95\% confidence
Fig. 2 Occurrence of acute/ delayed nausea and vomiting in HEC- and MEC-induced CINV. Proportion of patients with nausea and vomiting. Acute phase: $\leq 24 \mathrm{~h}$ from administration of emetogenic agents, delayed phase: $>24 \mathrm{~h}$ from administration

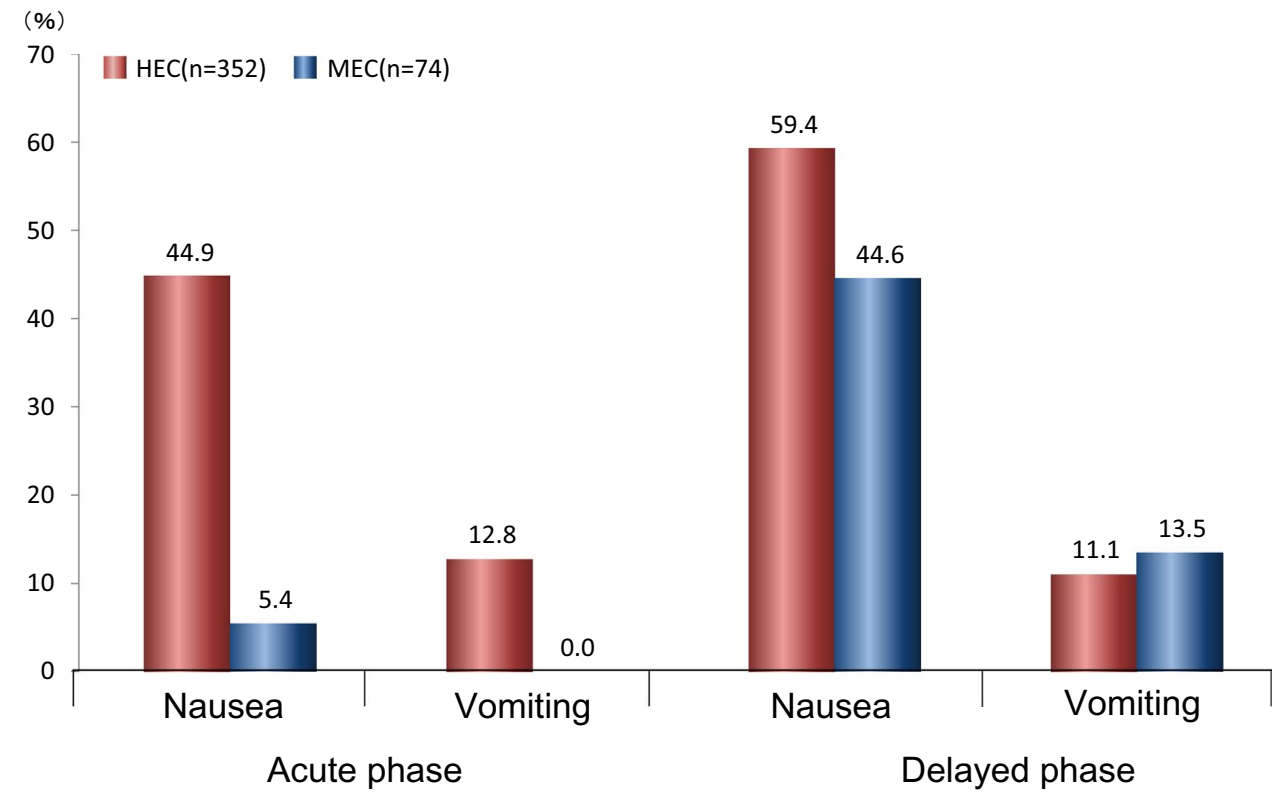




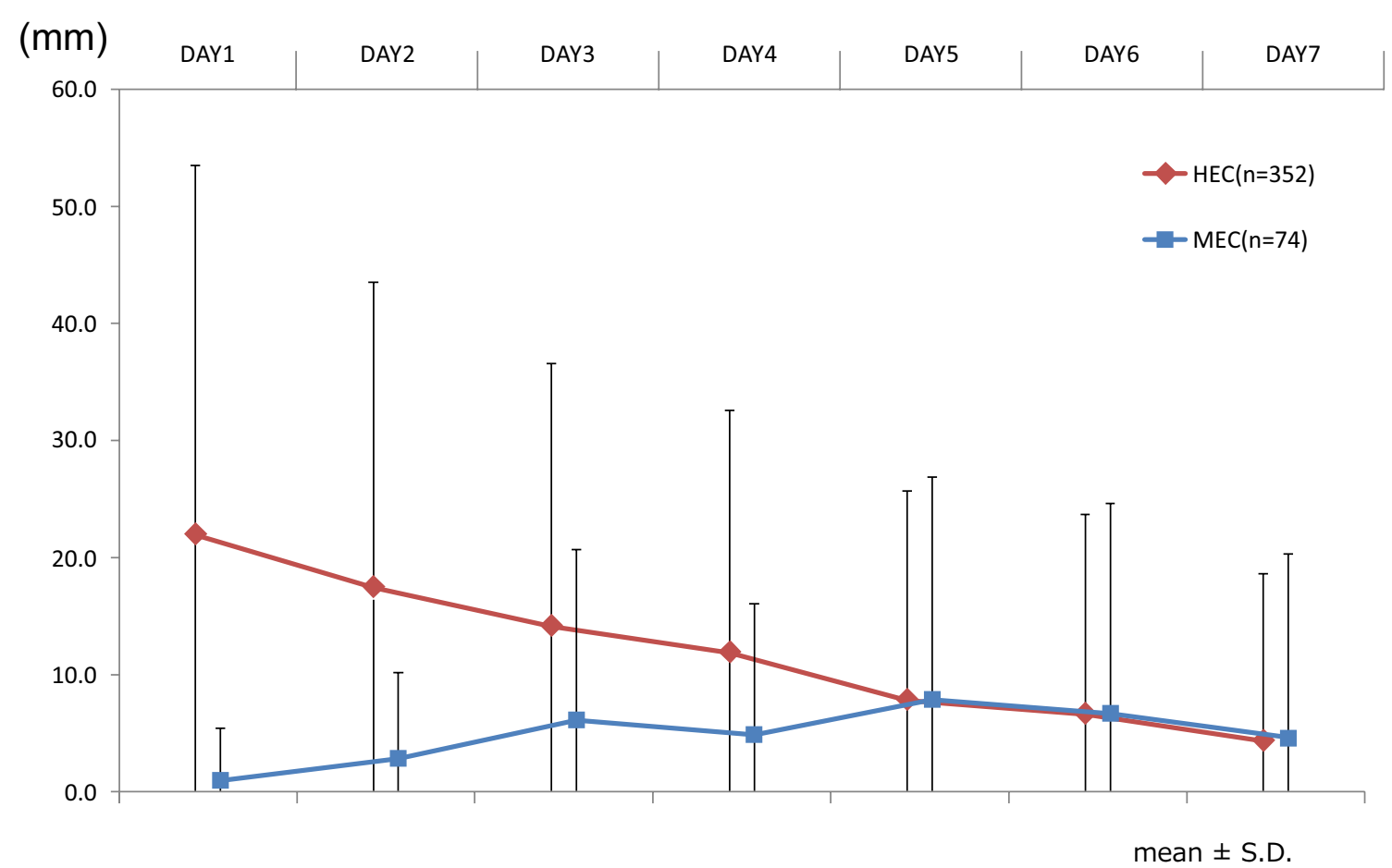

Fig. 3 VAS of nausea from day 1 to day 7. Visual analog scale (VAS) for the degree of nausea

interval $[\mathrm{CI}] 1.17-3.54 ; p=0.0115)$ were significantly correlated with nausea (Fig. 4c). Experience of hyperemesis gravidarum was also correlated with vomiting (OR 4.23; 95\% CI 2.08-8.61; $p<0.0001$ ) (Fig. 4d).

\section{Discussion}

Our study has demonstrated the actual status of CINV in patients with breast cancer in accordance with the current guidelines in Japan. Although the Hawthorne effect may have been present in this study (i.e., the treating physician might have paid more attention than in routine daily practice), high adherence to the guidelines deserves special mention. In the INSPIRE study conducted in the United States, only $57.3 \%$ of patients were treated with guideline-consistent antiemetic therapy, although adherence to antiemetic guidelines can significantly reduce the incidence of CINV after HEC and MEC [9]. In the PEER study conducted in Europe, guideline-consistent antiemetic therapy was administered to $55 \%$ and $46 \%$ of patients during the acute and delayed phases, respectively, and to $29 \%$ of patients throughout the overall study period (acute plus delayed phases); this was true despite the fact that again, adherence to antiemetic guidelines can significantly reduce the incidence of CINV [10].

Grunberg et al. reported that physicians and nurses markedly underestimated the incidence of delayed nausea and emesis after both HEC and MEC [11]. Our study consistently confirmed that it is difficult to accurately estimate whether a patient will develop CINV after administration of HEC or MEC. Therefore, we recommend to adhere to the

Table 2 Estimation of chemotherapy-induced nausea and vomiting (CINV) by medical staff compared with actual CINV

\begin{tabular}{|c|c|c|c|}
\hline & \multicolumn{3}{|c|}{ Estimation by medical staff $(n=426)$} \\
\hline & No CINV & CINV & Total \\
\hline \multicolumn{4}{|c|}{ Acute nausea kappa $=0.0958$} \\
\hline No & $68(16.0 \%)$ & $196(46.0 \%)$ & $264(62.0 \%)$ \\
\hline Yes & $23(5.4 \%)$ & $139(32.6 \%)$ & $162(38.0 \%)$ \\
\hline Total & $91(21.4 \%)$ & $335(78.6 \%)$ & $426(100 \%)$ \\
\hline \multicolumn{4}{|c|}{ Acute vomiting kappa $=0.0795$} \\
\hline No & $305(71.8 \%)$ & $75(17.6 \%)$ & $380(89.4 \%)$ \\
\hline Yes & $31(7.3 \%)$ & $14(3.3 \%)$ & $45(10.6 \%)$ \\
\hline Total & $336(79.1 \%)$ & $89(20.9 \%)$ & $425(100 \%)$ \\
\hline \multicolumn{4}{|c|}{ Delayed nausea kappa $=0.0237$} \\
\hline No & $49(11.5 \%)$ & $135(31.8 \%)$ & $184(43.2 \%)$ \\
\hline Yes & $59(13.8 \%)$ & $183(43.0 \%)$ & $242(56.8 \%)$ \\
\hline Total & $108(25.4 \%)$ & $318(74.6 \%)$ & $426(100 \%)$ \\
\hline \multicolumn{4}{|c|}{ Delayed vomiting kappa $=0.0092$} \\
\hline No & $312(73.2 \%)$ & $65(15.3 \%)$ & $377(88.5 \%)$ \\
\hline Yes & $40(9.4 \%)$ & $9(2.1 \%)$ & $49(11.5 \%)$ \\
\hline Total & $352(82.6 \%)$ & $74(17.4 \%)$ & $426(100 \%)$ \\
\hline
\end{tabular}

$C I N V$ chemotherapy-induced nausea and vomiting 
Fig. 4 Multivariate analysis of prognostic factors. a Multivariate analysis of prognostic factors for acute-phase nausea. Multivariate analysis showed that pregnancy experience and treatment with HEC were significantly correlated with developing acute-phase nausea. b Multivariate analysis of prognostic factors for acutephase vomiting. Younger age was the only factor which was significantly correlated with acute-phase vomiting. $\mathbf{c}$ Multivariate analysis of prognostic factors for delayed-phase nausea. Experience of motion sickness, younger age, a higher serum albumin concentration, and treatment with HEC were significantly correlated with delayed-phase nausea. d Multivariate analysis of prognostic factors for delayedphase vomiting. Experience of hyperemesis gravidarum was the only factor which was correlated with delayed-phase vomiting. Covariates included in the analyses were as follows; history of motion sickness (yes vs no), reproductive history (yes vs no), history of pregnancyrelated vomiting (yes vs no), alcohol intake history (yes vs no), age (continuous variable), hemoglobin (continuous variable), serum albumin concentration (continuous variable), number of prophylactic antiemetics (3 vs 2), use of 5HT3 antagonist (yes vs no), and HEC vs MEC a

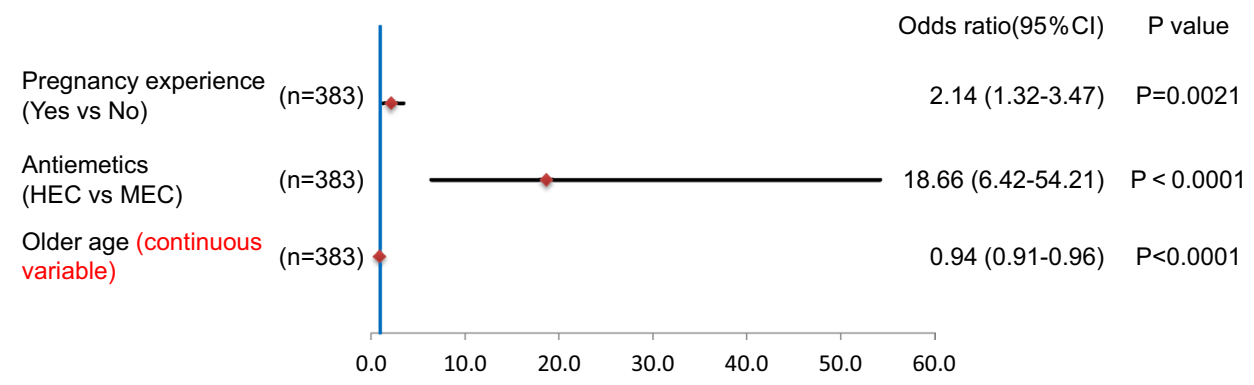

b

Older age (continuous variable)

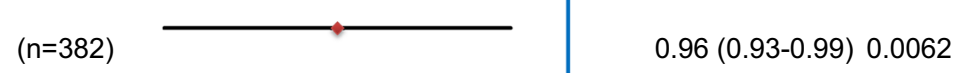

C

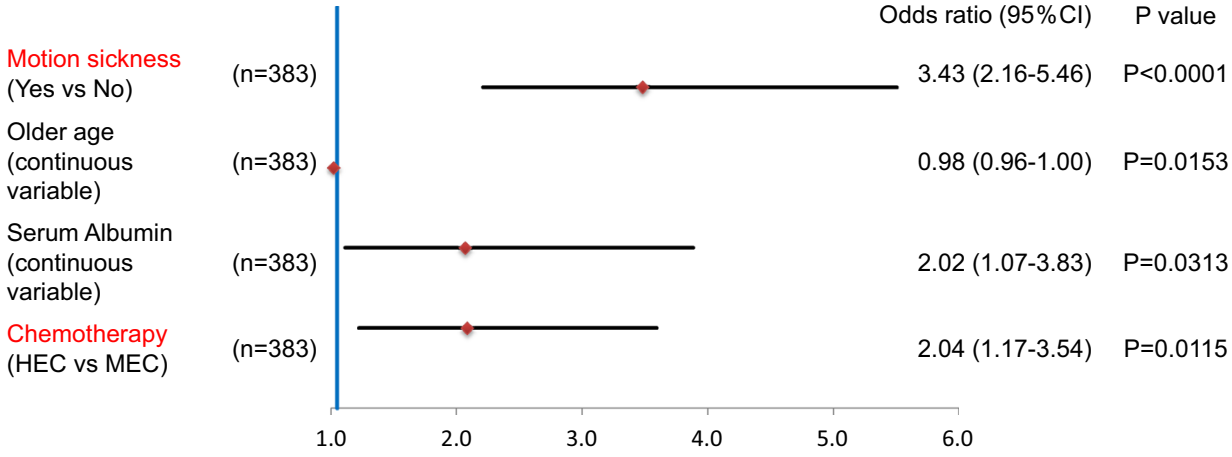

d

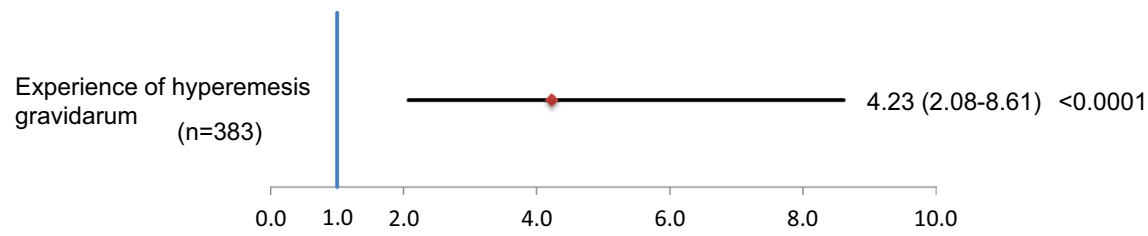

current guidelines and not to reduce antiemetics. Because CINV is one of the most clinically serious adverse reactions to chemotherapy, accurate estimation whether the patient will develop CINV or not is crucial; however, we found no sufficient predictors of CINV despite the fact that our study confirmed the previously reported risk factors for CINV. We found that younger age was a consistent risk factor for acute nausea, acute vomiting, and delayed nausea, and these findings are consistent with those of a previous study [12].

The proportion of patients who experienced nausea was higher in the delayed phase, which is consistent with a previous study [9-12]. However, the degree of the nausea was worst on the first day based on the VAS score. This is important information, because almost all recent investigations to improve the control of CINV have focused on 
delayed nausea and vomiting; in the present study, however, the patients suffered most from acute nausea, not delayed nausea. In future trials, we should evaluate VAS scores and be particularly aware of the degree of acute nausea.

In conclusion, the present study demonstrated high compliance to the CINV guidelines. Although underestimation of CINV by treating staff was resolved to some extent, accurately estimating CINV is still difficult. VAS scores revealed that acute nausea remains a clinically serious adverse reaction to HEC.

Acknowledgements The authors thank Etsuko Kumakawa, Yukimi Itoh, Noriko Ikoma, Noriko Gushima, and Kazuko Nakata for assisting with the registration and analysis of the collected data. We are grateful to Kazuo Tamura and Keisuke Aiba for their helpful advice as members of the Scientific Committee, and Masaki Kitajima, Yoshihiko Maehara, and Koichi Hirata as members of the Executive Council. The authors also thank all patients and investigators who participated in this study.

Funding The authors received a research grant from the Public Health Research Foundation.

\section{Compliance with ethical standards}

Conflict of interest Toshiaki Saeki has received speaker honorarium from Novartis and financial support for educational programs from Taiho Pharmaceutical Co., Ltd. and Eisai Co., Ltd. Yoichi Naito, Yuichiro Kai, Takashi Ishikawa, Tomoyuki Fujita, Kanou Uehara, Hiroyoshi Doihara, Shinya Tokunaga, Mototsugu Shimokawa, and Yoshinori Ito have declared no conflict of interest.

Ethical approval All procedures performed in studies involving human participants were in accordance with the ethical standards of the institutional and/or national research committee and with the 1964 Helsinki declaration and its later amendments or comparable ethical standards.

Informed consent Informed consent was obtained from all individual participants included in the study.

Open Access This article is distributed under the terms of the Creative Commons Attribution 4.0 International License (http://creativecommons.org/licenses/by/4.0/), which permits unrestricted use, distribution, and reproduction in any medium, provided you give appropriate credit to the original author(s) and the source, provide a link to the Creative Commons license, and indicate if changes were made.

\section{References}

1. U.S. Cancer Statistics Working Group. United States Cancer Statistics (2012) United States cancer statistics: 1999-2012 incidence and mortality web-based report. https://nccd.cdc.gov/USCS/topte ncancers.aspx. Accessed 20 Jan 2016.

2. Pruitt SL, Lee SJ, Tiro JA, et al. Residential racial segregation and mortality among black, white, and Hispanic urban breast cancer patients in Texas, 1995 to 2009. Cancer. 2015;121:1845-55.

3. Giordano SH, Buzdar AU, Smith TL, et al. Is breast cancer survival improving? Cancer. 2014;100:44-52.

4. Basch E, Prestrud AA, Hesketh PJ, et al. Antiemetics: American Society of Clinical Oncology clinical practice guideline update. J Clin Oncol. 2011;29:4189-98.

5. Roila F, Herrstedt J, Aapro M, et al. Guideline update for MASCC and ESMO in the prevention of chemotherapy- and radiotherapyinduced nausea and vomiting: results of the Perugia consensus conference. Ann Oncol. 2010;21(Suppl 5):232-43.

6. National Comprehensive Cancer Network. NCCN clinical practice guidelines in oncology 2012: antiemesis. http://www.nccn.org/ professionals/physician_gls/PDF/antiemesis.pdf. Accessed 20 Jan 2016.

7. Tamura K, Aiba K, Saeki T, et al. Testing the effectiveness of antiemetic guidelines: results of a prospective registry by the CINV Study Group of Japan. Int J Clin Oncol. 2015;20:855-65.

8. Takeuchi H, Saeki T, Aiba K, et al. Japanese Society of Clinical Oncology clinical practice guidelines 2010 for antiemesis in oncology: executive summary. Int J Clin Oncol. 2016;21:1-12.

9. Gilmore JW, Peacock NW, Gu A, et al. Antiemetic guideline consistency and incidence of chemotherapy-induced nausea and vomiting in US community oncology practice: INSPIRE Study. J Oncol Pract. 2014;10:68-74.

10. Aapro M, Molassiotis A, Dicato M, et al. The effect of guidelineconsistent antiemetic therapy on chemotherapy-induced nausea and vomiting (CINV): the Pan European Emesis Registry (PEER). Ann Oncol. 2012;23:1986-92.

11. Grunberg SM, Deuson RR, Mavros P, et al. Incidence of chemotherapy-induced nausea and emesis after modern antiemetics. Cancer. 2014;100:2261-8.

12. Tonato M, Roila F, Del Favero A. Methodology of antiemetic trials: a review. Ann Oncol. 1991;2:107-14.

Publisher's Note Springer Nature remains neutral with regard to jurisdictional claims in published maps and institutional affiliations. 